\title{
Publisher's Note: Nature of the many-body excitations in a quantum wire: Theory and experiment [Phys. Rev. B 93, 075147 (2016)]
}

O. Tsyplyatyev, A. J. Schofield, Y. Jin, M. Moreno, W. K. Tan, A. S. Anirban, C. J. B. Ford, J. P. Griffiths, I. Farrer, G. A. C. Jones, and D. A. Ritchie

(Received 9 March 2016; published 18 March 2016)

DOI: 10.1103/PhysRevB.93.119908

This paper was published online on 24 February 2016 with an omission of a data statement and a corresponding reference. The last paragraph before the Acknowledgments section should read as "Data associated with this work are available from [50]." The last reference should read as "[50] https://www.repository.cam.ac.uk/handle/1810/254193." The paper has been corrected as of 9 March 2016. The text and references are incorrect in the printed version of the journal. 